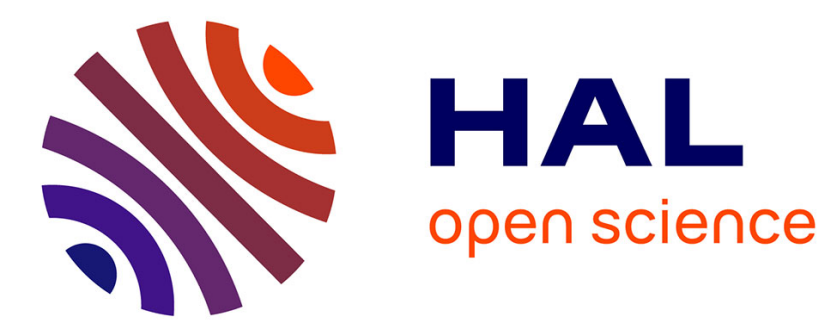

\title{
Escort entropies and divergences and related canonical distribution
}

Jean-François Bercher

\section{To cite this version:}

Jean-François Bercher. Escort entropies and divergences and related canonical distribution. Physics Letters A, 2011, 375 (33), pp.2969-2973. 10.1016/j.physleta.2011.06.057 . hal-00766631

\section{HAL Id: hal-00766631 \\ https://hal.science/hal-00766631}

Submitted on 18 Dec 2012

HAL is a multi-disciplinary open access archive for the deposit and dissemination of scientific research documents, whether they are published or not. The documents may come from teaching and research institutions in France or abroad, or from public or private research centers.
L'archive ouverte pluridisciplinaire HAL, est destinée au dépôt et à la diffusion de documents scientifiques de niveau recherche, publiés ou non, émanant des établissements d'enseignement et de recherche français ou étrangers, des laboratoires publics ou privés. 


\title{
Escort entropies and divergences and related canonical distribution
}

\author{
J.-F. Bercher \\ Université Paris-Est, LIGM, UMR CNRS 8049, ESIEE-Paris \\ 5 bd Descartes, 77454 Marne la Vallée Cedex 2, France
}

\begin{abstract}
We discuss two families of two-parameter entropies and divergences, derived from the standard Rényi and Tsallis entropies and divergences. These divergences and entropies are found as divergences or entropies of escort distributions. Exploiting the nonnegativity of the divergences, we derive the expression of the canonical distribution associated to the new entropies and a observable given as an escort-mean value. We show that this canonical distribution extends, and smoothly connects, the results obtained in nonextensive thermodynamics for the standard and generalized mean value constraints.
\end{abstract}

Keywords: Generalized Rényi and Tsallis entropies, $q$-gaussian distributions, Escort distributions PACS: 02.50.-r, 05.90.+m, 89.70.+c

\section{Introduction}

Rényi and Tsallis entropies extend the standard Shannon-Boltzmann entropy, enabling to build generalized thermostatistics, that include the standard one as a special case. The thermodynamics derived from Tsallis entropy, the nonextensive thermodynamics, has received a high attention and there is a wide variety of applications where experiments, numerical results and analytical derivations fairly agree with the new formalisms [1]. Some physical applications of the generalized entropies, - including statistics of cosmic rays, defect turbulence, optical lattices, systems with long-range interactions, superstatistical systems, etc., can be found in the recent review [2] and references therein. In the extended thermodynamics, it has been found particularly useful to use generalized moments $[3,4]$. These moments are computed with respect to a deformed version of the density at hands, which is called its escort distribution [5, 6]. Actually, several type of constraints $[7,8,9]$ have been used in order to derive the canonical distributions: a first type of constraints is expressed as standard linear mean values while a second type of constraints is given as generalized escort mean values. In the two cases, the related canonical distributions are expressed as a $q$-Gaussian distribution, but with two opposite exponents, and both solutions reduce to a standard Gaussian distribution in the $q=1$ case. These $q$-Gaussian distributions can exhibit a power-law behavior, with a remarkable agreement with experimental data, see for instance $[1,10,11]$, and references therein. These distributions are also analytical solutions of actual physical problems, e.g. [12, 13, 14]. There has been numerous discussions regarding the choice of a 'correct' form of the constraints, either as a standard mean or an escort-average, and on the connections between the solutions and associated thermodynamics. In particular, dualities and equivalences between the two settings have been described.

This is precisely the context of the present Letter, where we propose a simple connection between these different formulations and between the related canonical distributions. More specifically, we suggest a simple way to combine the originally distinct concepts of entropy and escort distributions into a single two-parameter $(a, \lambda)$ entropy. Then, we propose to look at an associated extended maximum entropy problem. This approach includes the two aforementioned formulations as special cases. Exploiting the nonnegativity of the associated divergence, we derive the expression of the canonical distribution for an observable given as an escort-mean value. We show

Email address: jf.bercher@esiee.fr(J.-F. Bercher) 
that this canonical distribution extends, and smoothly connects, the results obtained in nonextensive thermodynamics for the standard and generalized mean value constraints.

We begin by recalling the context and definitions in section 2 . Then, the combined escort divergences and entropies are introduced and discussed in section 3. The related maximum entropy problem and its solution are described in section 4. Finally, in section 5, we illustrate the results in the case of a two-level system.

\section{Context and definitions}

\subsection{Main definitions}

Let us recall that if $f$ and $g$ are two probability densities defined with respect to a common measure $\mu$, then for a parameter $q>0$, called the entropic index, the Tsallis information divergence is defined by

$$
D_{q}^{(T)}(f \| g)=\frac{1}{q-1}\left(\int f(x)^{q} g(x)^{1-q} \mathrm{~d} \mu(x)-1\right),
$$

and similarly, the Rényi information divergence is defined by

$$
D_{q}^{(R)}(f \| g)=\frac{1}{q-1} \log \int f(x)^{q} g(x)^{1-q} \mathrm{~d} \mu(x),
$$

provided, in both cases, that the integral is finite. By l'Hospital's rule, both Tsallis and Rényi information divergences reduce to the Kullback-Leibler information in the case $q=1$. Associated to these divergences, we obtain the entropies when $g(x)$ is uniform with respect to $\mu$. For $q \neq 1$,

$$
S_{q}[f]=\frac{1}{1-q}\left(\int f(x)^{q} \mathrm{~d} \mu(x)-1\right),
$$

is the Tsallis entropy, and

$$
H_{q}[f]=\frac{1}{1-q} \log \int f(x)^{q} \mathrm{~d} \mu(x),
$$

is the Rényi entropy. In both cases, we have $S_{1}[f]=H_{1}[f]=-\int f \log f \mathrm{~d} \mu(x)$, the Shannon entropy, for $q=1$. For the Lebesgue measure, the definitions reduces to the standard differential entropies. Finally, in the discrete case, the continuous sum is replaced by a discrete one which extends on a subset $\mathcal{D}$ of integers, and $\mu$ is a counting measure, usually taken as uniform. We will also denote

$$
M_{q}[f]=\int f(x)^{q} \mathrm{~d} \mu(x)
$$

the integral of the density raised to the power $q$.

The escort distributions used in nonextensive statistics are defined as follows. If $f(x)$ is an univariate probability density, then its escort $f_{a}(x)$ of order $a, a \geq 0$, is given by

$$
f_{a}(x)=\frac{f(x)^{a}}{\int f(x)^{a} \mathrm{~d} \mu(x)},
$$

provided that $M_{a}[f]=\int f(x)^{a} \mathrm{~d} \mu(x)$ is finite. Given that $f_{a}(x)$ is the escort of $f(x)$, we see that $f(x)$ is itself the escort of order $1 / a$ of $f_{a}(x)$. Accordingly, the (absolute) generalized $a$-moment of order $p$ is defined by

$$
m_{p, a}[f]=\int|x|^{p} f_{a}(x) \mathrm{d} x=\frac{\int|x|^{p} f(x)^{a} \mathrm{~d} \mu(x)}{\int f(x)^{a} \mathrm{~d} \mu(x)} .
$$

Of course, standard moments are recovered in the case $a=1$.

In the context of nonextensive statistical mechanics, the parameter $a$ is taken equal to the parameter $q$ of the $q$-entropy. We shall also point out that the 'deformed' information measure like the Rényi entropy (3) and the escort distribution (5) are originally two distinct concepts, as indicated here by the different notations $a$ and $q$. There is an interesting discussion on this point in [15]. It is a contribution of the present paper to indicate that it is still possible to adopt two different values for these parameters. 


\subsection{The maximum entropy problems}

In nonextensive thermostatistics, the maximum $q$-entropy distributions, which are identified as $q$-gaussian distributions, can be obtained using a constraint expressed as a classical mean value; but it has also been found adequate to use constraints given as escort-mean values, with the same index $q$ as in the entropy. This is discussed in [7] or in [3, 4]. In the first case, the maximum entropy problem stands as the variational problem

$$
H_{q}^{(1)}(m)=\max _{f}\left\{H_{q}[f]: m_{p, 1}[f]=m \text { and } m_{0,1}[f]=1\right\}
$$

which consists in finding a distribution with maximum Rényi entropy on the set of all probability distributions with a fixed moment of order $p: m_{p, 1}[f]=m$. The value of the maximum entropy obtained for a given moment is denoted $H_{q}(m)$ - the use of the square brackets and parenthesis distinguishes between the functions of the state and the functions of the observable. Similarly, for a constraint given as an escort-mean value, we have

$$
H_{q}^{(q)}(m)=\max _{f}\left\{H_{q}[f]: m_{p, q}[f]=m \text { and } m_{0,1}[f]=1\right\} .
$$

Note that although the problems above have been written for the Rényi entropy, the very same problems can be written for Tsallis entropy; actually the optimum distributions are identical and the optimum values of both maximum entropy problems are easily related.

In the case of the standard mean constraint, the density that achieves the maximum entropy is given by the generalized $q$-Gaussian

$$
f(x)=\frac{1}{Z(\beta)}\left(1-(q-1) \beta|x|^{p}\right)_{+}^{\frac{1}{q-1}}
$$

while in the case of the escort mean constraint, the solution has an exponent with opposite sign

$$
f(x)=\frac{1}{Z(\beta)}\left(1-(1-q) \beta|x|^{p}\right)_{+}^{\frac{1}{1-q}} .
$$

In both cases, we used the notation $(x)_{+}=\max \{x, 0\}$, and $\beta$ is a positive parameter chosen so as to satisfy the constraint. The limit case $q \rightarrow 1$ gives $f(x) \propto \exp \left(-\beta|x|^{p}\right)$. The partition functions $Z(\beta)$ used to normalize $f(x)$ as a density will be given below.

\subsection{Dualities}

There has been a great deal of discussions concerning the relationships between the two problems (7) and (8) with standard and escort mean value constraints, and between their solutions (9) and (10). Dualities between the solutions have been introduced: the $q \rightarrow 2-q$ transformation [16, 17] enables to obtain the density (10) from the maximization of the entropy $S_{2-q}[f]$ subject to standard constraints rather than the maximization of $S_{q}[f]$ subject to escort generalized constraints. The $q \rightarrow 1 / q$ duality rests on the duality between the original density and its escort: as already noted, if $f_{q}$ is the escort of order $q$ of $f$, then $f$ is itself the escort of order $1 / q$ of $f_{q}$. This has been mentioned in [7], the related duality discussed in [18] and in [19]. It is very easy to obtain the $q \rightarrow 1 / q$ duality as the consequence of the symmetry above for escort distributions: it suffices to note that for the Rényi entropy, we always have the equality $H_{\frac{1}{q}}\left[f_{q}\right]=H_{q}[f]$. Therefore, noting that we also have $m_{p, q}[f]=m_{p, 1}\left[f_{q}\right]$, we see that the problem

$$
H_{q}^{(q)}(m)=\max _{f}\left\{H_{q}[f]: m_{p, q}[f]=m \text { and } m_{0,1}[f]=1\right\},
$$

is exactly equivalent to

$$
H_{q}^{(q)}(m)=\max _{f}\left\{H_{\frac{1}{q}}\left[f_{q}\right]: m_{p, 1}\left[f_{q}\right]=m \text { and } m_{0,1}[f]=1\right\}=H_{\frac{1}{q}}^{(1)}(m)
$$

where the maximum entropy is obtained for the density (10). Therefore, it is always possible to swap the standard and generalized constraints, provided that entropic index $q$ is changed into $1 / q$. We shall also mention that these dualities have been linked to combinatorial considerations in [20]. 


\section{The $(a, \lambda)$ divergences and entropies}

In complement to the dualities mentioned above, we will show that there is a continuum of $q$-Gaussians, solutions of an extended maximum entropy problem, that smoothly connects the problems (7) and (8) and their solutions (9) and (10). The basic idea is to mix the concepts of $q$-entropies and escort distributions into a single quantity. This leads us to a simple extension of the Rényi (Tsallis) information divergence and entropy to a two parameters case. Interestingly, the generalized $(a, \lambda)$-Rényi information divergence that emerges in (14) has been mentioned [21, eq. (49)] as a possible extension of a new class of $(\alpha, \beta)$-divergences, generalizing a family of Gamma-divergences recently introduced in [22].

Definition 1. Given two parameters $a$ and $\lambda>0$ and two densities $f$ and $g$, we call $(a, \lambda)$-Rényi information divergence the standard Rényi information divergence with index $q=a / \lambda$ between the escort distributions like (5), of order $\lambda, f_{\lambda}$ and $g_{\lambda}$, associated to $f$ and $g$ :

$$
D_{a, \lambda}^{(R)}(f \| g)=D_{\frac{a}{\lambda}}^{(R)}\left(f_{\lambda} \| g_{\lambda}\right) .
$$

In the developed form, this gives

$$
D_{a, \lambda}^{(R)}(f \| g)=\frac{1}{a-\lambda} \log \frac{\left[\int f(x)^{a} g(x)^{\lambda-a} \mathrm{~d} \mu(x)\right]^{\lambda}}{\left[\int f(x)^{\lambda} \mathrm{d} \mu(x)\right]^{a}\left[\int g(x)^{\lambda} \mathrm{d} \mu(x)\right]^{\lambda-a}},
$$

provided that the different integrals exist.

Similarly, we call $(a, \lambda)$-Tsallis information divergence the quantity

$$
D_{a, \lambda}^{(T)}(f \| g)=\frac{1}{a-\lambda}\left(\frac{\left[\int f(x)^{a} g(x)^{\lambda-a} \mathrm{~d} \mu(x)\right]^{\lambda}}{\left[\int f(x)^{\lambda} \mathrm{d} \mu(x)\right]^{a}\left[\int g(x)^{\lambda} \mathrm{d} \mu(x)\right]^{\lambda-a}}-1\right)
$$

provided that the different integrals exist.

These definitions include the Rényi (Tsallis) information divergence $D_{a}^{(.)}(f \| g)$ in the case $\lambda=1$. In the case $\lambda=a$, again by l'Hospital's rule, (14) becomes $D_{\lambda, \lambda}^{(.)}(f \| g)=D\left(f_{\lambda} \| g_{\lambda}\right)$, the Kullback-Leibler divergence between the escort distributions of order $\lambda, f_{\lambda}$ and $g_{\lambda}$, associated to $f$ and $g$. Finally, in the case $a=\lambda=1$, the generalized Rényi (Tsallis) information divergence reduces to the standard Kullback-Leibler divergence.

Associated to this $(a, \lambda)$-Rényi information divergences, we can also define a two parameters Rényi (Tsallis) entropy.

Definition 2. Given $a$ and $\lambda>0$ and a density $f$, we call $(a, \lambda)$-Rényi entropy the entropy of order $q=a / \lambda$ of the escort $f_{\lambda}$ :

$$
H_{a, \lambda}[f]=H_{\frac{a}{\lambda}}\left[f_{\lambda}\right]=\frac{1}{\lambda-a} \log \left[\int f(x)^{a} \mathrm{~d} \mu(x)\right]^{\lambda}\left[\int f(x)^{\lambda} \mathrm{d} \mu(x)\right]^{-a},
$$

provided that the different integrals exist. Similarly, the $(a, \lambda)$-Tsallis entropy is given by

$$
S_{a, \lambda}[f]=S_{\frac{a}{\lambda}}\left[f_{\lambda}\right]=\frac{1}{\lambda-a}\left(\left[\int f(x)^{a} \mathrm{~d} \mu(x)\right]^{\lambda}\left[\int f(x)^{\lambda} \mathrm{d} \mu(x)\right]^{-a}-1\right),
$$

Again, for $\lambda=1$ we get the standard Rényi (Tsallis) entropy of order $a$, for $a=1$ we get the standard Rényi (Tsallis) entropy of order $\lambda$, for $a=\lambda$ it reduces to the Shannon entropy of the escort distribution, and to the standard Shannon entropy in the case $a=\lambda=1$.

In the following, we will need the fact that the divergence $D_{a, \lambda}^{(.)}(f \| g)$ is always nonnegative:

Proposition 3. if $f$ and $g$ are two densities such that the involved integrals are finite, with a and $\lambda>0$, then $D_{a, \lambda}^{(\cdot)}(f \| g) \geq 0$, with equality if and only if $f=g$.

Proof. Obviously, once we have realized that $D_{a, \lambda}^{(.)}(f \| g)$ in (14) is the Rényi (Tsallis) divergence between escort distributions, this is a direct consequence of the nonnegativity of the Rényi (Tsallis) divergence, which follows from Jensen inequality. Alternatively, this could also be derived from the Hölder inequality. 


\section{The $(a, \lambda)$ maximum entropy problem}

With the previous definitions at hands, we can now consider the following extended maximum entropy problem:

$$
H_{a, \lambda}(m)=\max _{f}\left\{H_{a, \lambda}[f]: m_{p, a}[f]=m \text { and } m_{0,1}[f]=1\right\}
$$

which includes the previous problems as particular cases. The usual procedure for handling such variational problem is the technique of Lagrangian multipliers, e.g. [7, 8]. However, even though the objective functional is strictly concave (here for $a \leq \lambda$ ), the constraints set is not convex and the uniqueness and nature of the maximum can not be guaranteed. The situation is still more involved in the continuous setting considered here which requires the results from calculus of variations [23,24]. Therefore, we propose to derive the solution as consequence of the nonnegativity of $D_{a, \lambda}(f \| g)$, without recourse to the technique of Lagrange multipliers. We obtain that the density $f(x)$ that achieves the maximum in the right hand side of (18) is a generalized Gaussian, as given by the following proposition.

Proposition 4. The density that achieves the maximum of the $(a, \lambda)$-Rényi or Tsallis entropy, subject to the escort mean constraint $m_{p, a}[f]=m$, is the generalized Gaussian

$$
G_{\beta}(x)= \begin{cases}\frac{1}{Z(\beta)}\left(1-(\lambda-a) \beta|x|^{p}\right)^{\frac{1}{\lambda-a}} & \text { for } \lambda \neq a \\ \frac{1}{Z(\beta)} \exp \left(-\beta|x|^{p}\right) & \text { if } \lambda=a\end{cases}
$$

where $\beta$ is a positive parameter. The partition function $Z(\beta)$ is given by

$$
\begin{aligned}
& Z(\beta)=\frac{2}{p}(\beta)^{-\frac{1}{p}} \times \\
& \begin{cases}(a-\lambda)^{-\frac{1}{p}} B\left(\frac{1}{p},-\frac{1}{(\lambda-a)}-\frac{1}{p}\right) & \text { for } a>\lambda>a-p \\
(\lambda-a)^{-\frac{1}{p}} B\left(\frac{1}{p}, \frac{1}{(\lambda-a)}+1\right) & \text { for } \lambda>a \\
\Gamma\left(\frac{1}{p}\right) & \text { and if } \lambda=a\end{cases}
\end{aligned}
$$

where $B(x, y)$ is the Beta function. Finally, the a-moment of order $p$ is given by

$$
m_{p, a}\left[G_{\beta}\right]= \begin{cases}\frac{1}{\beta p(\lambda-a)\left(\frac{a}{\lambda-a}+\frac{1}{p}+1\right)} & \text { for } \lambda-a \neq 0 \\ \frac{1}{\beta p a} & \text { for } \lambda=a .\end{cases}
$$

For $\lambda>a$, the density has a compact support, while for $\lambda \leq a$ it is defined on the whole real axis and behaves as a power distribution for $|x| \rightarrow \infty$.

Proof. The expressions of the partition function and of the $a$-moment are obtained by direct calculation. For the main result, let us consider the generalized Gaussian (19), and denote $A(\beta)=1 / Z(\beta)$ the inverse of the partition function. We directly have

$$
\begin{aligned}
\int f^{a} G_{\beta}^{\lambda-a} \mathrm{~d} \mu(x) & =A(\beta)^{\lambda-a} M_{a}[f] \times \int\left(1-(\lambda-a) \beta|x|^{p}\right)_{+} \frac{f^{a}}{M_{a}[f]} \mathrm{d} \mu(x) \\
\leq & A(\beta)^{\lambda-a}\left(1-(\lambda-a) \beta m_{p, a}[f]\right) M_{a}[f],
\end{aligned}
$$

where we have exhibited the escort distribution $f_{a}$, where $m_{p, a}[f]$ denotes the generalized $a$-moment, and where the inequality in (23) results from the fact that for $\lambda>a$, the support of $\left(1-(\lambda-a) \beta|x|^{p}\right)_{+}$can be smaller than the support of $f$. From (23) we also immediately get with $f=G_{\beta}$ that

$$
M_{\lambda}\left[G_{\beta}\right]=A(\beta)^{\lambda-a}\left(1-(\lambda-a) \beta m_{a, p}\left[G_{\beta}\right]\right) M_{a}\left[G_{\beta}\right] .
$$


Therefore, for all distributions $f$ with a given $a$-moment $m_{p, a}[f]=m$ and for $\beta$ such that the generalized Gaussian has the same moment $m_{p, a}\left[G_{\beta}\right]=m$, then the combination of (23) and (24) gives

$$
\int f^{a} G_{\beta}^{\lambda-a} \mathrm{~d} \mu \leq \frac{M_{\lambda}\left[G_{\beta}\right]}{M_{a}\left[G_{\beta}\right]} M_{a}[f] .
$$

Finally, the $(a, \lambda)$-Rényi divergence can be expressed as

$$
D_{a, \lambda}^{(R)}\left(f \| G_{\beta}\right)=\log \left(\frac{\left[\int f^{a} G_{\beta}^{\lambda-a} \mathrm{~d} \mu(x)\right]^{\lambda}}{M_{\lambda}[f]^{a} M_{\lambda}\left[G_{\beta}\right]^{\lambda-a}}\right)^{\frac{1}{a-\lambda}} \leq \log \left(\frac{M_{a}\left[G_{\beta}\right]^{-\lambda} M_{\lambda}\left[G_{\beta}\right]^{a}}{M_{a}[f]^{-\lambda} M_{\lambda}[f]^{a}}\right)^{\frac{1}{a-\lambda}}=H_{a, \lambda}\left(G_{\beta}\right)-H_{a, \lambda}(f),
$$

by the definitions (14) and (16) of the divergence and entropy. By the nonnegativity of the divergence, we obtain that

$$
H_{a, \lambda}\left(G_{\beta}\right) \geq H_{a, \lambda}(f)
$$

for all distributions $f$ with a given $a$-moment $m_{p, a}[f]=m_{p, a}\left[G_{\beta}\right]=m$, and with equality iff $f=G_{\beta}$. Using the $(a, \lambda)$-Tsallis divergence rather than Rényi's, the same result also directly follows for the $(a, \lambda)$-Tsallis entropy.

From this result, we see that the problem (18) and its solution indeed interpolates between the maximum $q$-entropy problem with standard constraint (7) and with generalized $q$-moment constraint (8).

- For $a=q$ and $\lambda=1$, the $(q, 1)$-Rényi entropy is the standard Rényi entropy with index $q$, whose maximum subject to a $q$-moment constraint is attained for the generalized Gaussian (10) with exponent $1 /(1-q)$.

- For $a=1$ and $\lambda=q$, the $(1, q)$-Rényi entropy is the Rényi entropy with index $q$, whose maximum subject to a standard moment constraint is the generalized Gaussian (9) with exponent $1 /(q-1)$.

It shall be also mentioned that we still get a $q \rightarrow 1 / q$ duality result in this setting. From the definition (16), we always have the identity $H_{a, \lambda}[f]=H_{\frac{a}{\lambda}}\left[f_{\lambda}\right]=H_{\frac{\lambda}{a}}\left[f_{a}\right]$. In the other hand, the generalized $a$-moment $m_{p, a}[f]$ can be considered as the standard moment of density $f_{a}, m_{p, a}[f]=m_{p, 1}\left[f_{a}\right]$ but also as the escort moment of order $a / \lambda$ of the density $f_{\lambda}$, i.e. $m_{p, a}[f]=m_{p, \frac{a}{\lambda}}\left[f_{\lambda}\right]$. Therefore, the problem (18) can be recasted in the two equivalent forms

$$
H_{a, \lambda}(m)=\max _{f}\left\{H_{\frac{\lambda}{a}}\left[f_{a}\right]: m_{p, 1}\left[f_{a}\right]=m \text { and } m_{0,1}[f]=1\right\}
$$

which is a problem with respect to the density $f_{a}$ under a standard mean constraint, or

$$
H_{a, \lambda}(m)=\max _{f}\left\{H_{\frac{a}{\lambda}}\left[f_{\lambda}\right]: m_{p, \frac{a}{\lambda}}\left[f_{\lambda}\right]=m \text { and } m_{0,1}[f]=1\right\}
$$

which is a problem with respect to $f_{\lambda}$ with a generalized moment constraint.

\section{The case of a two-level system}

Finally, we close this paper with the example of a two-level system, with eigenenergies 0 and 1 . Although the general $(a, \lambda)$ maximum entropy problem (18) usually do not lead to closed-form expressions for the entropies $H_{a, \lambda}(m)$, see e.g. [25], we still can get an explicit solution for the simple two-level system. For this system, the measure at hands charges the two levels, with $\mu=\delta_{0}+\delta_{1}$ where $\delta_{x}$ denotes the Dirac mass at $x$. Then, the maximum entropy distribution is the discrete distribution with a probability $p$ for the excited state and $(1-p)$ for the ground state, with

$$
p=\frac{(1+\beta)^{\frac{1}{\lambda-a}}}{1+(1+\beta)^{\frac{1}{\lambda-a}}} .
$$


In turn, the $(a, \lambda)$-entropy is then given by

$$
H_{a, \lambda}\left[G_{\beta}\right]=\frac{1}{\lambda-a} \log \left[p^{a}+(1-p)^{a}\right]^{\lambda}\left[p^{\lambda}+(1-p)^{\lambda}\right]^{-a},
$$

and the internal energy, $m$, which is computed with respect to the escort distribution of order $a$ is simply

$$
m=\frac{p^{a}}{p^{a}+(1-p)^{a}} .
$$

With a little algebra, it is possible to express $p$ as a function of $m$ :

$$
p=\frac{m^{\frac{1}{a}}}{m^{\frac{1}{a}}+(1-m)^{\frac{1}{a}}},
$$

and then we can get the expression of the $(a, \lambda)$-entropy:

$$
H_{a, \lambda}(m)=\frac{a}{a-\lambda} \log \left[m^{\frac{\lambda}{a}}+(1-m)^{\frac{\lambda}{a}}\right] .
$$

From this expression, it is easy to derive an expression of the inverse temperature as the derivative with respect to $m$, or the expression of the related heat capacity. We also see that the subsequent entropy only depends on the ratio $\lambda / a$. Finally, for $\lambda=a$, l'Hospital's rule gives

$$
H_{a, a}(m)=-m \log [m]-(1-m) \log (1-m),
$$

which is the Fermi-Dirac entropy.

\section{Conclusions}

In this Letter, we have suggested a possible extension of the standard $q$-divergences and entropies by considering a combination of the concepts of $q$-divergences and of escort distributions. This leads to two-parameter information measures that recover the classical ones as special cases, and coincide with some recently proposed measures [21]. Further work should examine the general properties of these information measures. We have introduced a general maximum entropy problem and derived the expression of the canonical distribution maximizing the $(a, \lambda)$-entropy under a generalized $a$-moment constraint. This was obtained as a consequence of the nonnegativity of the related information divergence, without recourse to the technique of Lagrange multipliers. This canonical distribution is a two-parameter version of the generalized $q$-Gaussian distribution, extending the versatility of this distribution. It includes the standard solutions of the nonextensive thermostatistics as particular cases. Actually, the proposed generalized maximum entropy problem includes the standard approaches of nonextensive thermostatistics and provides a continuum of problems and solutions between them. In addition, this approach suggests that it is possible to adopt different indexes for the $q$-entropy and for the escort-mean constraint.

[1] C. Tsallis, Introduction to Nonextensive Statistical Mechanics, 1st Edition, Springer, 2009.

[2] C. Beck, Contemp. Physics 50 (2009) 495.

[3] S. Abe, G. B. Bagci, Phys. Rev. E 71 (2005) 016139.

[4] C. Tsallis, A. R. Plastino, R. F. Alvarez-Estrada, J. Math. Phys. 50 (2009) 043303.

[5] A. Chhabra, R. V. Jensen, Phys. Rev. Lett. 62 (1989) 1327.

[6] C. Beck, F. Schloegl, Thermodynamics of Chaotic Systems, Cambridge University Press, 1993.

[7] C. Tsallis, R. S. Mendes, A. R. Plastino, Physica A 261 (1998) 534. 
[8] S. Martínez, F. Nicolás, F. Pennini, A. Plastino, Physica A 286 (2000) 489.

[9] G. L. Ferri, S. Martinez, A. Plastino, J. Stat. Mech. (2005) P04009.

[10] H. J. Hilhorst, G. Schehr, J. Stat. Mech. Theor. Exp. 2007 (2007) P06003.

[11] C. Vignat, A. Plastino, Physica A 388 (2009) 601.

[12] E. Lutz, Phys. Rev. A 67 (2003) 051402.

[13] V. Schwämmle, F. D. Nobre, C. Tsallis, European Phys. J. B 66 (2008) 537.

[14] A. Ohara, T. Wada, J. Phys. A Math. Gen. 43 (2010) 035002.

[15] F. Pennini, A. Plastino, G. Ferri, Physica A 383 (2007) 782.

[16] F. Baldovin, A. Robledo, Phys. Rev. E 69 (2004) 045202.

[17] T. Wada, A. Scarfone, Phys. Lett. A 335 (2005) 351.

[18] G. A. Raggio, cond-mat/9908207, (1999).

[19] J. Naudts, Chaos Solitons Fractals 13 (2002) 445.

[20] H. Suyari, T. Wada, Physica A 387 (2008) 71.

[21] A. Cichocki, S. Cruces, S. ichi Amari, Entropy 13 (2011) 134.

[22] H. Fujisawa, S. Eguchi, J. Multivar. Anal. 99 (2008) 2053.

[23] I. M. Gelfand, S. V. Fomin, Calculus of Variations, Dover Publications, 2000.

[24] M. Giaquinta, S. Hildebrandt, Calculus of Variations: The Lagrangian formalism, Springer, 1996.

[25] J. F. Bercher, Inf. Sci. 178 (2008) 2489. 